\title{
Performance Analysis of Circular 8-QAM Constellation with MMSE Equalizer for OFDM System Using USRP
}

\section{Muh. Alfan Taufiqurrahman, I Gede Puja Astawa, Amang Sudarsono}

\author{
Graduate School of Engineering Technology \\ Department of Electrical Engineering \\ Politeknik Elektronika Negeri Surabaya \\ PENS Campus, Jalan Raya ITS, Sukolilo 60111, Indonesia \\ E-mail: alfantaufiqurrahman@pasca.student.pens.ac.id, \{puja, amang\}@pens.ac.id
}

\begin{abstract}
Bandwidth is very important in communication system, and it is a limited resource. In order to save the limited bandwidth resource, high order $\mathrm{M}$-ary modulation is widely employed in modern communication and broadcasting systems. In Orthogonal Frequency Division Multiplexing (OFDM), fading environment lead to a loss of orthogonality between the subcarriers. In this paper, we present the performance analysis of circular 8-Quadrature Ampilutude Modulation (QAM) constellation for Orthogonal Frequency Division Multiplexing (OFDM) system. We also combine the system with Minimum Mean Square Error (MMSE) equalizer to mitigate the effect of Inter Symbol Interference (ISI). Then, all of this system is implemented practically using Universal Software Radio Peripheral (USRP). The performance of circular 8-QAM and MMSE equalizer is evaluated by comparing with other 8-QAM modulation models such as circular-Zero Forcing (ZF), star-ZF, Square-ZF, Star-MMSE, and Square-MMSE. The performance of circular 8-QAM with MMSE equalizer is better than the other combinations. Bit Error Rate (BER) graph shows that the performance of circular-MMSE is better than star-MMSE. The performance improvement using circular-MMSE is about $1.6 \%$. The performance will decrease when the distance is increased. The performance of this system is greatly affected by the distance between transmitter and receiver.
\end{abstract}

Keywords: OFDM, Circular-8QAM, USRP, MMSE Equalizer

\section{INTRODUCTION}

Currently, the development of wireless communication technology is very fast, many users need higher data rate communication, so that it needs large bandwidth to support the development of wireless communication. Bandwidth is determined by the symbol rate rather than bit rate [1]. Bandwidth is very important thing in wireless communication system, and it is very limited, so that it must be used efficiently. For saving the limited bandwidth resource, high order M-ary modulation is widely employed in 
modern communication and broadcasting systems. In M-ary modulation, several bits can be represented in one symbol, by this way it can increase the data rate communication. However, the higher index of modulation is very sensitive to noise effect, not only White Gaussian Noise, but also Phase Noise which is caused by many factors in transmitter and receiver side. To combat this problem, many researchers research about the best constellation diagram to mitigate noise variation.

Nowadays, square constellation diagram, is widely used in wireless communication system. The square type is used because the square type's symbol distances are same for all constellation points. Several types of M-ary modulation are widely assigned to different subcarriers based to the bits. Among the candidates, 8-QAM is the one that presents many possible signal constellations with different performance when distinct symbol distances and amplitudes are considered. Therefore, it needs an investigation and research to determine the best type or model of constellation diagram for 8QAM.

There are several types of constellation model for QAM, such as circular and star. The circular constellation has been widely used in telecommunication, especially in broadcasting system. The circular constellation is used because circular QAM has smaller amplitude fluctuation compared with rectangular or square QAM. [2-3]. Nadal et al have conducted research about several types of constellation diagram for 8-QAM such as star, circular, rectangular and square. The paper compare the performance of four types of constellation diagram for 8-QAM. The circular constellation has a better performance than the square constellation. However, the potential of circular constellation to combat phase noise effect is overlooked. Based on that fact, the higher index of circular constellation can be effectively used to improve bandwidth efficiency in high speed communication. These have not been applied widely because higher index of modulation is very sensitive to disturbance of noise.

In wireless communication, the Gaussian noise, Doppler effects, multipath fading cause bad affect in transmission. Therefore, today OFDM is widely applied on wireless communication systems, because OFDM can combat these problem [4]. The performance of OFDM depends on modulation technique which used on the system. One of problem that degrades the performance of modulation is phase noise. Phase noise can turn the distribution symbols in constellation diagram to be not accurate, so that it makes the demodulation process cannot work very well. Another solution for this bad effect is by applying the equalizer technique.

The equalizer technique in OFDM is a digital filter that provides approximate inverse of channel frequency response. The purpose of this equalizer is to mitigate the effect of ISI (Inter symbol Interference) and to decrease the probability of error that occurs without suppression of ISI. There are several equalizer techniques which is commonly used in OFDM system, such as ZF and MMSE. The potential of these equalizers have been 
widely research. The previous research about different equalization technique in OFDM system is shown that Bit Error Rate (BER) can be improved by using equalizer [5]. Furthermore, the MMSE outperforms than ZF equalizer because MMSE not only mitigate the effect of ISI, but also minimize the noise power.

In this paper, we propose the combination of circular 8-QAM with MMSE equalizer technique in OFDM system. The performance of this combination is evaluated by bit error rate percentage. The performance analysis has been done in real time communication between transmitter and receiver by using Software Defined Radio (SDR) and USRP as front end.

\section{RELATED WORKS}

Several researches about OFDM system have been taken. An OFDM system which is using Binary Phase Shift Keying (BPSK) and Quadrature Phase Shift Keying (QPSK) modulation and implemented in USRP by Pollet et al [4]. The result of that research is a good performance achieved by using QPSK modulation rather than using BPSK modulation. An experimental research of several 8-QAM constellation on OFDM system are investigated by Nadal et al and Li et al [2,3]. The research investigate 8-QAM constellation model such as square, circular, star, rectangular and how this model impact the system performance. The main problem in wireless communication is noise in channel environment. To combat this noise on OFDM system, several researches about equalizer technique have been conducted. The performance of OFDM with different equalizer technique such as ZF equalizer and MMSE has been done in the simulation program by Archa et al, and Abirami et al [56]. The result of these papers are the equalizer technique, ZF and MMSE, can combat the effect of ISI and proved by BER graph that the performance is better than without equalizer technique.

\section{ORIGINALITY}

This paper presents the performance analysis of OFDM system combine with circular 8-QAM and MMSE equalizer technique. As an improvement of the research before, we add signal to noise ratio estimation for the MMSE equalizer algorithm. This system is successfully built in real implementation using Lab View platform and USRP-N2920 as front end. Reliability system is investigated based on bit error percentage in receiver side. The reliability of the system is investigated based on bit error percentage in receiver side. The information that is sent to the receiver side in this system is text file using an OFDM system. Furthermore, in the receiver side, a MMSE equalizer is used to mitigate the effect of Inter symbol Interference (ISI).

\section{SYSTEM DESIGN}

The experiment of this research is to implement the OFDM transceiver system with 8-QAM modulation and MMSE equalizer technique. The OFDM transceiver system is modified using circular 8-QAM modulation. Then, the 
performance of these circular 8-QAM in OFDM system combined with MMSE equalizer is observed in real implementation system using NI-USRP 2920 device.

\subsection{Orthogonal Frequency Division Multiplexing (OFDM)}

OFDM is one of multicarrier modulation technique, which divided data into several parallel streams. OFDM concept has subcarrier that orthogonality, from this concept OFDM allow the spectrum of each subchannel to overlap each other without interfering with it. In the effect of this, is seen as the required bandwidth that greatly reduced by removing guard bands and allowing signal to overlap. It can reduce interference between subcarrier and save spectrum bandwidth. OFDM technique is stream the data and split it into parallel streams, so that when the original bit rate is $R$, then the bit rate in each parallel line is $\mathrm{R} / \mathrm{M}$ where $\mathrm{M}$ is the number of parallel lines (number of sub-carrier). The spectrum of OFDM is shown on figure 1.

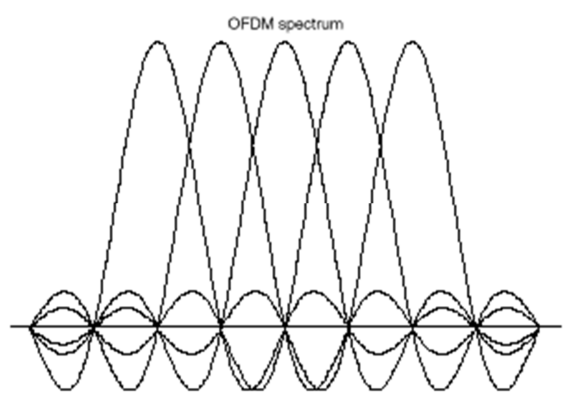

Figure 1. OFDM Spectrum

In the block of digital modulation, the data on each symbol is mapped to a particular phase based on modulation used such as 4-QAM, 8QAM, etc. Each one the phase is assigned a unique pattern of binary bit. Usually, each phase encodes an equal number of bits.

The Inverse Fast Fourier Transform (IFFT) processes convert frequency domain data into time domain signal. Prior to IFFT mapping zero-padding is performed to adjust the IFFT bit size of length. Zero padding is used because the number of subcarriers may be less than bit size. After IFFT process, the parallel stream is converted to serial stream. Each stream contains one carrier out of group of orthogonal carrier. The cyclic prefix (CP) process is added to cyclic extension of an OFDM symbol to eliminate the ISI effect on original OFDM symbol. The common of cyclic prefix length is $1 / 4$ of the length of symbol. The cyclic prefix adds time over head decreasing the overall spectral efficiency of the system.

Received signals on receiver contain signal plus noise. In the receiver side do the reverse in contrast to the OFDM transmitter. The first process in receiver is serial output channel is converted into parallel stream then removing cyclic prefix. In this process, the receiver must know the location of cyclic prefix. OFDM system needs synchronization in the receiver side to find 
the beginning of each symbol correctly. Synchronization parameters include finding the right time delay, frequency deviation and phase shift of each symbol in the subcarrier. These parameters can be determined by adding redundancy in some of the subcarriers which transmitted. The redundancy is called as pilot symbol or preamble. The parameters are known by looking pilot signal from data received and will be calculated for synchronization Then Fast Fourier Transform (FFT) process to convert on time domain. After that data processed by using equalizer to mitigate the noise effect, and demodulation before converting into the real data. The overall pictures of the system working OFDM transmitter and receiver are indicated by figure 2[7].

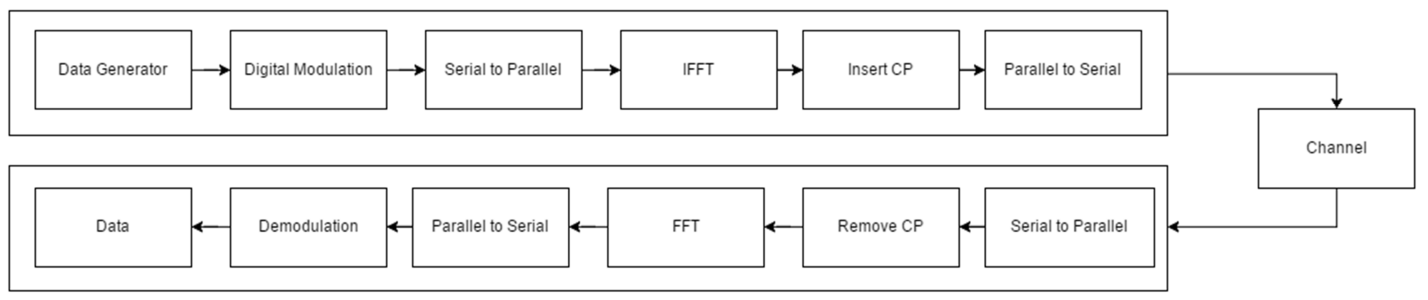

Figure 2. Block Diagram of OFDM Transceiver System

\subsection{Circular 8-QAM (Quadrature Amplitude Modulation)}

Quadrature amplitude modulation (QAM) is both analog and digital modulation scheme. It conveys two analog message signals, or two digital bit streams, by changing (modulating) the amplitudes of two carrier waves, using the amplitude-shift keying (ASK) digital modulation scheme or amplitude modulation (AM) analog modulation scheme. The two carrier waves, usually sinusoids, are out of phase with each other by $90^{\circ}$ and are thus called quadrature carriers or quadrature components. QAM is used extensively as a modulation scheme for digital telecommunication systems. Arbitrarily high spectral efficiencies can be achieved with QAM by setting suitable constellation size, limited only by the noise level and linearity of the communications channel. QAM is generally used. Because the QAM constellation points are much more disperse than PSK constellation points and the distances between the constellation points are much bigger with the same ary. So QAM modulation could provide a better transmission performance.QAM signal wave forms may be expressed as equation (1).

$S_{m}(t)=A_{m} g(t) \cos 2 \pi f_{c} t-A_{m s} g(t) \sin 2 \pi f_{c} t$

Where $A_{m c}$ and $A_{m s}$ are the information-bearing signal amplitudes of the quadrature carriers and $g(t)$ is the signal pulse. The vector representations of this waveform is

$S_{m}=\left[A_{m c} \sqrt{\frac{1}{2}} \varepsilon_{g} A_{m s} \sqrt{\frac{1}{2}} \varepsilon_{g}\right]$ 
Where $\varepsilon_{g}$ is the energy of the basic signal pulse $g(t)$. M-QAM constellations can be constructed in many different ways, and they have different capacity and error characteristics. Although rectangular, circle, and star signal constellations are common in practice; a certain kind of constellation can be designed to achieve the best communication performance, under some specific premises [12].

Next, let us consider $\mathrm{M}=8 \mathrm{QAM}$, in this case there are many possible signals constellation. All of possible constellations consist of two amplitudes and it has a minimum distance between signal points. The coordinates $\left(A_{m c}\right.$, $A_{m s}$ ) for each signal points. The average transmitted signal power is

$P_{a v}=\frac{1}{M} \sum_{m=1}^{M}\left(A_{m c}^{2}+A_{m s}^{2}\right)$

Where $\left(A_{m c}, A_{m s}\right)$ are the coordinates of the signal points, normalized by $A$. [13]

In regular circular M-QAM each ring is equally spaced by distance and has four sets of symbols, which are $90^{\circ}$ apart from one another. The rings are classified as odd and even rings. Without lossing of generality, it is assumed that the odd rings have their symbols positioned on in-phase (real) and quadrature phase (imaginer). Let denote the number of bits in a symbol, then the total number of symbols can be determined by $M=2^{n}$. Moreover, the total number of rings required to accommodate $M$ symbols is calculated as $2^{n-2}$ [14]

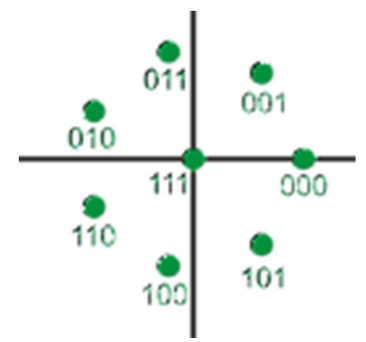

Figure 3. Example of Circular QAM

\subsection{Minimum Mean Square Error (MMSE) Equalizer}

The problem of wireless communication system is multipath fading effect that caused received signal effected ISI. The receiver side employes equalizer technique to mitigate the ISI effect in OFDM. Equalizer is a filter that uses approximate inverse of channel frequency response or channel estimation. ZF equalizer technique is simple equalizer in OFDM system to reduce of ISI effects. MMSE estimator is a method in which it minimizes the Mean Square Error (MSE). The characteristic of MMSE equalizer it does not usually eliminate the effect of ISI totally, but instead of minimizes the total power of the noise. Firsty, let assume that signal received can be written below on equation, that $\mathrm{H}$ be a channel matrix, and $\mathrm{x}$ be an unknown random variable and $\mathrm{n}$ be a noise received [5].

$Y=H \cdot x+n$ 
To solve equation above, channel matrix $(\mathrm{H})$ must be known earlier. MMSE equalizer is enhancement from $\mathrm{ZF}$ equalizer technique, which the $\mathrm{ZF}$ equalizer can be written mathematically as follows:

$W=\left(H^{H} \cdot H\right)^{-1} \cdot H^{H}$

In MMSE equalizer, there is signal to noise ratio component (No), therefore the MMSE equalizer can be written mathematically as follows [15].

$W=\left(H^{H} \cdot H+N o \cdot I\right)^{-1} \cdot H^{H}$

\subsection{Proposed Circular 8-QAM Constellation with MMSE Equalizer}

This paper explains about the performance of 8-QAM modulation with MMSE equalizer for OFDM system using USRP device in the laboratory scale. In this section, we will describe about method, topology, and performance measurement. One of the main requirements in communication system is bandwidth. The usage of bandwidth is very important and bandwidth is limited, so bandwidth must be used efficiently. In order to save the limited bandwidth resource, OFDM system is widely applied in modern communication and broadcasting systems. However, OFDM system needs the modulation method to modulate the signal. One of modulation types, QAM modulation, is commonly used with this system. Furthermore in wireless communication system, there are many problems can influence the performance, one of the problems is multipath fading. The bad effect of this problem can create ISI in OFDM system. Therefore in OFDM system also need equalization method to mitigate the effect of ISI.

In this research, we implement an OFDM transceiver system which use 8-QAM modulation, especially circular QAM modulation, and it also use equalization technique MMSE to mitigate the effect of ISI. In previous sections, it have mentioned that the main goal of this research to analyze the performance of circular QAM and performance of MMSE equalization technique.

In the transmitter side, there are several processes occurred. The process starts by determining the input of this system. In this research, we uses text file as an input. The next process is to convert the data into bit before modulation process. Bit streams processed by symbol mapper with appropriate modulation used. In this case, we adopt circular 8-QAM modulation technique. In the next step is core of OFDM process such as IFFT, and insert CP. The output from OFDM process is OFDM symbols that have been ready to be sent to receiver. The overall process in transmitter is depicted on figure 4.

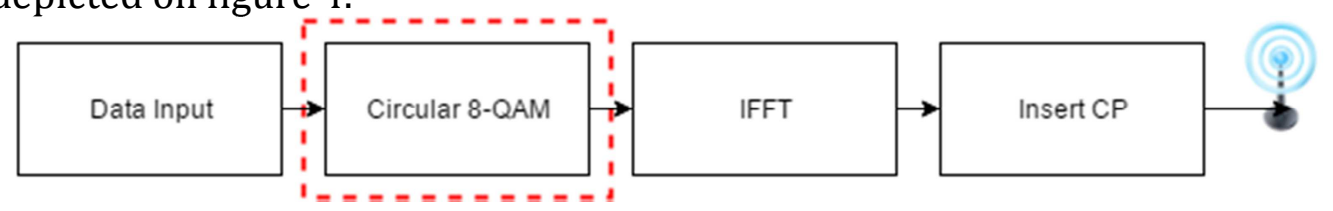

Figure 4.Block Diagram of Proposed Transmitter 
In the receiver side the opposite process of the transmitter side is occurred. The overall process in receiver is depicted on figure 5.

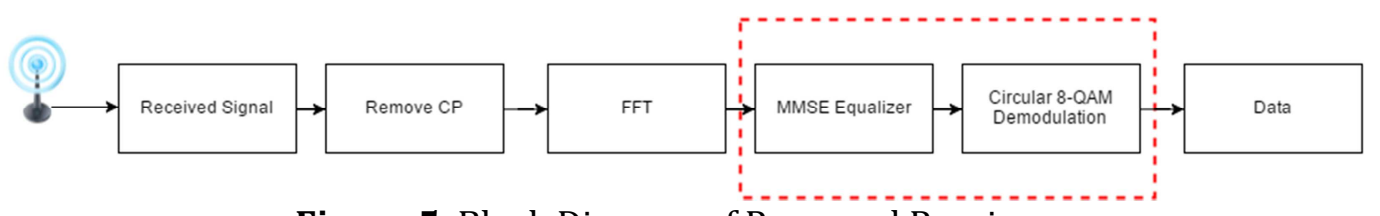

Figure 5. Block Diagram of Proposed Receiver

After data has been received in receiver, then data is extracted to remove CP. After, the CP is removed, the FFT process is occurred to transform the data back to frequency domain. In previous section, it has been mentioned in wireless communication that there are noise and multipath fading effect. Therefore in receiver side, there is equalization process before data demodulation process. The main goal of equalization process is to mitigate the bad effect of wireless communication. In this system we adopt MMSE as an equalizer. The process of MMSE equalizer is depicted on figure 6.

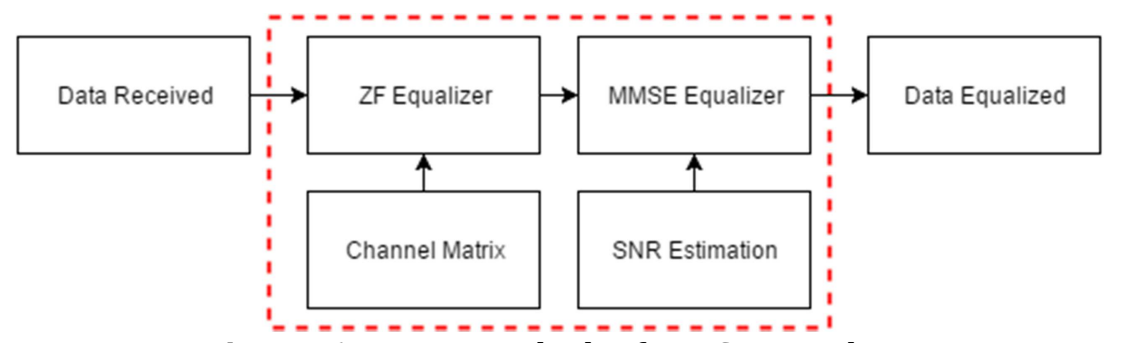

Figure 6. Diagram Block of MMSE Equalizer

MMSE equalizer process is begun with data which are already converted into frequency domain by FFT process. In previous section, it have been mentioned that MMSE is enhancement version of ZF equalizer. Therefore, the output from ZF equalizer is an input for MMSE equalizer. MMSE equalizer is not only mitigate the ISI but also minimize noise power, therefore in MMSE there is SNR estimation process. The output from this equalizer process are the equalized data that will be processed by demodulation process. In this system, we uses circular demodulation process similar with transmitter side modulation technique. The final output from receiver is text file.

In this system, OFDM transmitter with circular QAM modulation transmitted by USRP 1 as transmitter. On other side, OFDM receiver, circular QAM demodulation and MMSE equalizer is considered as a receiver in USRP 2.

\section{IMPLEMENTATION}

This section describes about system implementation. There are two main systems, transmitter and receiver. Both of OFDM transmitter and receiver system are implemented on USRP-N2920 using LabVIEW software platform. The first activity is configuring the USRP for transmitter. In this 
process includes determining the IP address for USRP transmitter, determining the signal source such as carrier frequency, antenna gain and active antenna.

The next activity is implementing an OFDM transmitter system. OFDM transmitter system in general is depicted in the figure 7.

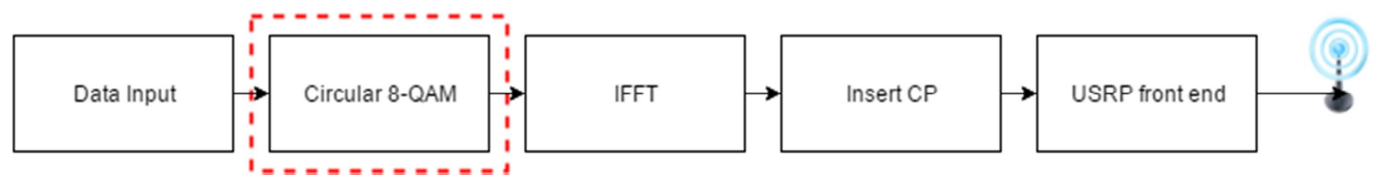

Figure 7. Transmitter Implementation on USRP

In this research, we use text file as data input. Data will convert into bit stream 0 and 1 before going to the next process. The bit stream is processed by mapper symbol using appropriate modulation. In this research, we used 8QAM modulation with circular constellation. After symbol mapper, the next process is IFFT process to convert the signal to frequency domain. Before the data is sent through wireless channel, the data is added with $1 / 4$ cyclic prefix by duplicating the last point of symbol and placed at the beginning of packet. The last process is to transmit the signals by using USRP as front end.

In the other side, it is begun by configuring the USRP for receiver. In this process includes determining the IP address for receiver, determining gain antenna, and active antenna. The next process is implementing an OFDM receiver system. In this research, we add one block for equalization process before demodulation process. OFDM receiver system is shown in the figure 8 .

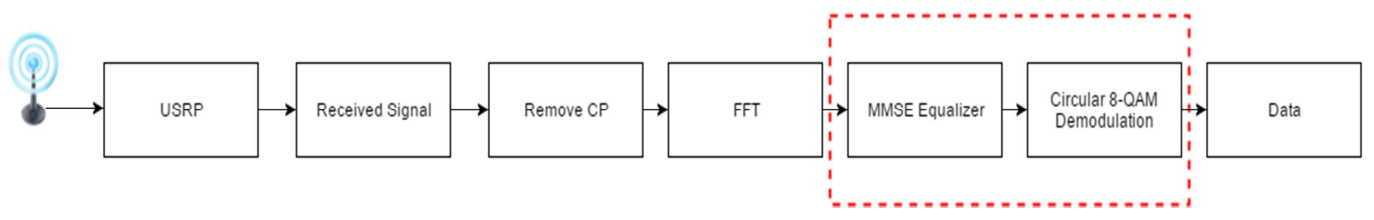

Figure 8.Receiver Implementation on USRP

The first process in the receiver side is to receive signal that has been transmitted by transmitter. The received signal includes data signal and noise, it refers to equation (4). The next step is separated the signal from the $\mathrm{CP}$. Then the FFT process is occurred to convert the signal into frequency domain. Before the demodulation process, the signal is processed in an equalization block. In this research, we use MMSE equalizer. In the previous section have been described the goal of equalization block is to mitigate the effect of ISI. The output of this equalized data.

\section{EXPERIMENT AND ANALYSIS}

In this section, we investigate the performance of OFDM transceiver with combination circular 8-QAM constellation and MMSE equalizer using USRP as front end and present the experimental results to confirm our 
proposed. This experiment uses single input and single output system. We involve two PC and two USRP as transmitter and receiver. The topology of this research showed on figure 9 .

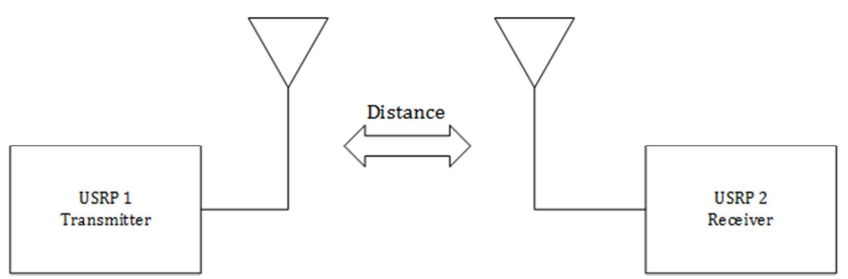

Figure 9. Experiment and Measurement Topology

To support our work required some parameters. In this research, we try to mimic IEEE 802.11.a standard. We use 52 numbers of subcarrier therefore, the total data transmitted will be 64. We use 8-QAM modulation and demodulation. We use $915 \mathrm{MHz}$ as the center frequency. The overall of our parameter is shown in table 1.

Table 1. Measurement Parameters

\begin{tabular}{|c|c|}
\hline Parameters & Value \\
\hline Number of Subcarrier & 52 \\
\hline Number of FFT & 64 \\
\hline Cyclic Prefix (CP) ratio & $1 / 4$ \\
\hline Modulation & $8-\mathrm{QAM}$ \\
\hline Center Frequency & $915 \mathrm{MHz}$ \\
\hline Number of Transmitter & $1 \mathrm{Antenna}$ \\
\hline Number of Receiver & $1 \mathrm{Antenna}$ \\
\hline Distance Variation (m) & $2 \mathrm{~m}, 5 \mathrm{~m}$, and $7 \mathrm{~m}$ \\
\hline Equalizer & $\mathrm{ZF} \mathrm{and} \mathrm{MMSE}$ \\
\hline Channel & Indoor Channel (Laboratory) \\
\hline Signal to Noise Ratio (SNR) & $50 \mathrm{~dB}$ \\
\hline Dimension Laboratory & $7 \mathrm{~m} \mathrm{x} \mathrm{7m}$ \\
\hline
\end{tabular}

Our implementation is built in LabVIEW program. We implement our proposed scheme in indoor channel. Our experiment scheme is shown in figure 10. In this work we investigate the performance of modulation and the performance MMSE equalizer with bit error percentages with distance variation of 2,5 , and 7 meters between transmitter and receiver. 


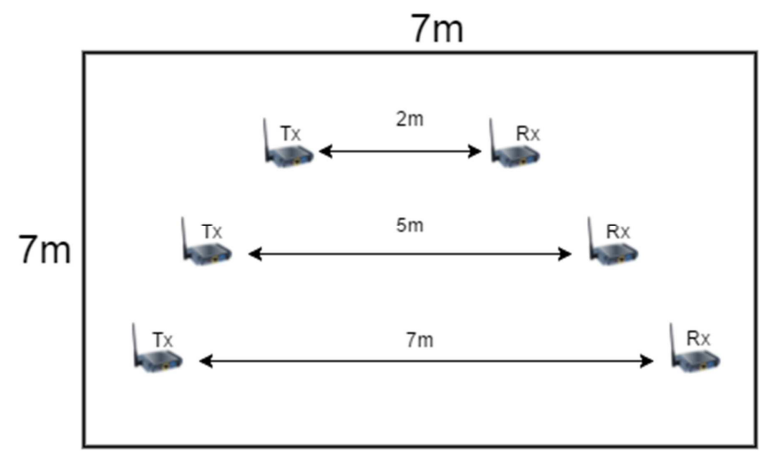

Figure 10. Experimental Distance between Transmitter and Receiver

\subsection{Measurement for Square Constellation with ZF equalizer}

We investigate the combination of square constellation for 8-QAM modulation with $\mathrm{ZF}$ equalizer. This measurement uses distance variation of $2 \mathrm{~m}, 5 \mathrm{~m}$, and $7 \mathrm{~m}$ between transmitter and receiver. In transmitter and receiver we implement square constellation for 8-QAM modulation, and we add ZF equalizer block in receiver. The first measurement investigates the constellation in receiver. From this measurement, we get three constellations for three distance variation. The figure 11 shows the constellation diagram with different distance. As the result, the constellation is more scattered for the distance above $2 \mathrm{~m}$. The second measurement investigates the performance of this combination. To support this measurement, we send text as a data input through our system, and the output of receiver is equalized data. The bit error performance is gotten by comparing equalized data with data input sent. From this experiment we get bit error percentages of $5 \%$ for $2 \mathrm{~m}$ distance, $9.8 \%$ for $5 \mathrm{~m}$, and $15 \%$ for $7 \mathrm{~m}$ distance from scale of $100 \%$.

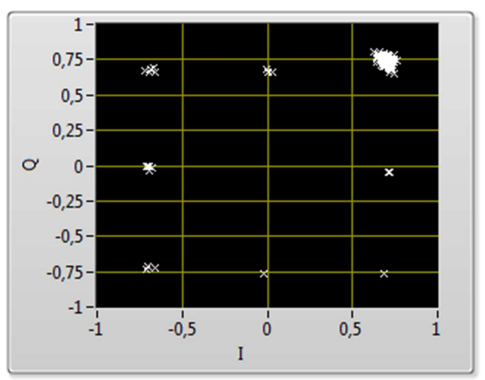

(a)

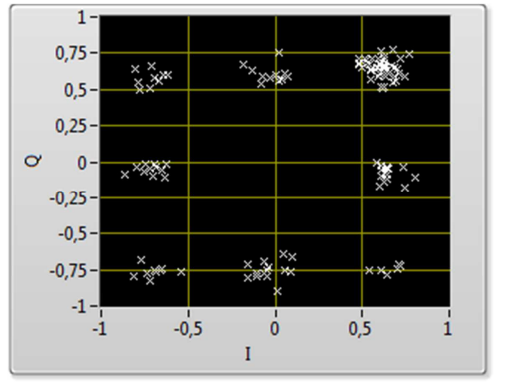

(b)

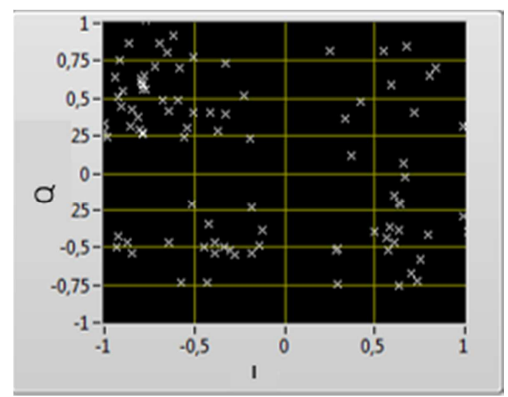

(c)

Figure 11. Square Constellation with ZF Equalizer (a) 2m (b) $5 \mathrm{~m}$ (c) $7 \mathrm{~m}$ 


\subsection{Measurement for Square Constellation with MMSE equalizer}

In this section, we apply and measure the combination of square constellation with MMSE equalizer. We implement circular 8-QAM modulation and square 8-QAM modulation in both transmitter and receiver, and also implement MMSE equalizer in receiver. MMSE equalizer is one level above from the $\mathrm{ZF}$ equalizer as mentioned in the previous section. The first measurement investigates the constellation diagram in receiver with the variation of distance. The figure 12 shows the constellation diagram with different distance. When using $2 \mathrm{~m}$ distance is gotten the best result constellation, while the distance above $2 \mathrm{~m}$, the constellation is more scattered. To investigate the performance of MMSE equalizer, we sent text as a data input through our system. The output of receiver is equalized data and compared with data input. From this experiment, we get bit error percentages $4.2 \%$ for $2 \mathrm{~m}$ distance, $9 \%$ for $5 \mathrm{~m}$ distance, and $14 \%$ for $7 \mathrm{~m}$ distance from scale of $100 \%$.

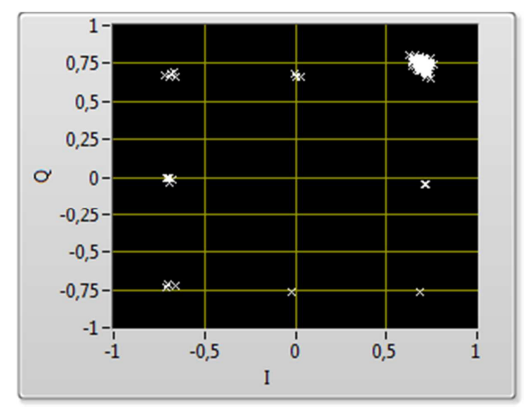

(a)

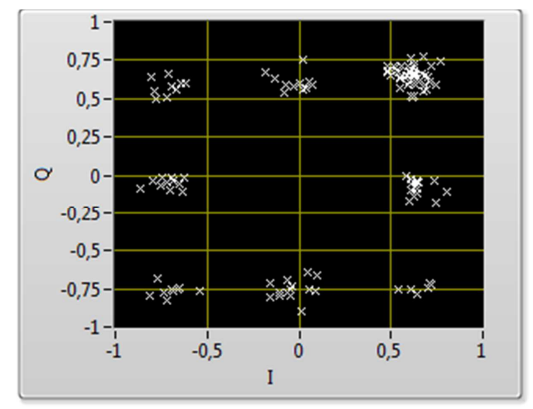

(b)

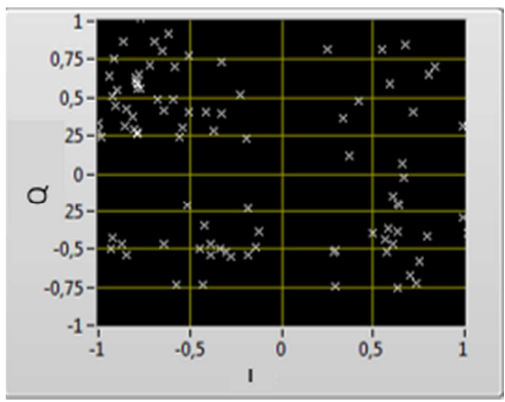

(c)

Figure 12. Square Constellation with MMSE Equalizer (a) 2m (b) $5 \mathrm{~m}$ (c) $7 \mathrm{~m}$

\subsection{Measurement for Star Constellation with ZF equalizer}

We investigate the combination of star constellation with ZF equalizer. There are two measurements. In this measurement, we implement star constellation and 8-QAM modulation in transmitter and receiver side, and also add $\mathrm{ZF}$ equalizer in receiver. The first measurement observes the constellation diagram of this combination in receiver with distance $2 \mathrm{~m}, 5 \mathrm{~m}$, and $7 \mathrm{~m}$ between transmitter and receiver. The figure 13 shows the 
constellation diagram of this combination with distance variation. The constellation on $5 \mathrm{~m}$, and $7 \mathrm{~m}$ is more scattered than the constellation on $2 \mathrm{~m}$ distance. To investigate the performance of this combination, we sent text as a data input through our system. The output of receiver is equalized data and compared with data input sent. From this experiment, we get the bit error percentage of $3.8 \%$ for $2 \mathrm{~m}$ distance, $8 \%$ for $5 \mathrm{~m}$ distance, and $14 \%$ for $7 \mathrm{~m}$ distance from scale of $100 \%$.

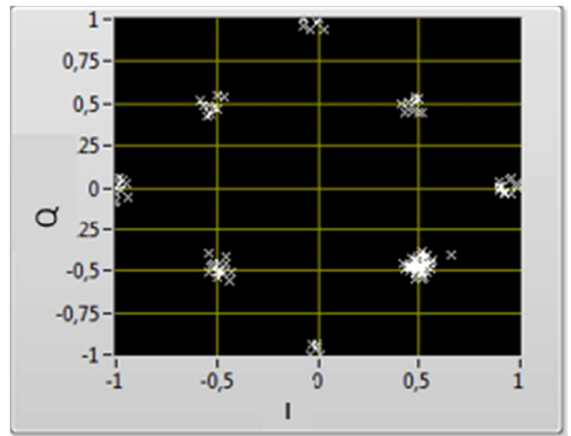

(a)

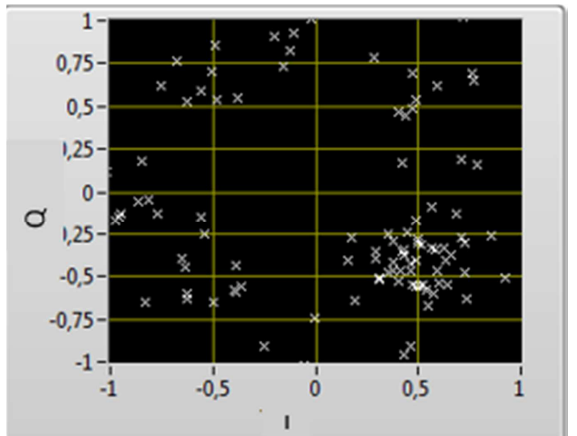

(b)

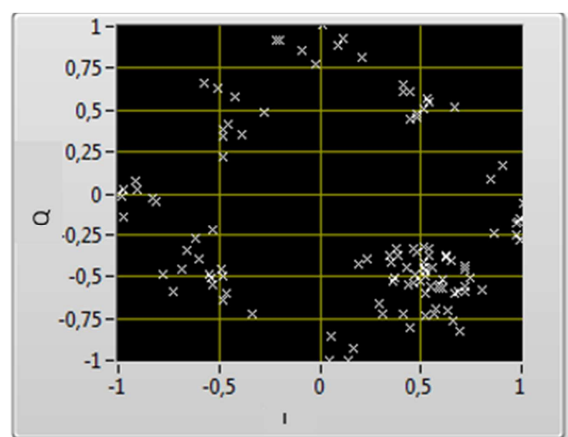

(c)

Figure 13. Star Constellation with ZF Equalizer (a) $2 \mathrm{~m}$ (b) $5 \mathrm{~m}$ (c) $7 \mathrm{~m}$

\subsection{Measurement for Star Constellation with MMSE equalizer}

We investigate the combination of star constellation with MMSE equalizer. In this section, we implement 8-QAM modulation and star constellation both transmitter and receiver, and also implement MMSE equalizer in the receiver. The first measurement observes the constellation diagram of this combination with $2 \mathrm{~m}, 5 \mathrm{~m}$, and $7 \mathrm{~m}$ distance between transmitter and receiver. The figure 14 shows constellation diagram of this combination with distance variation. The next measurement investigates the performance of this combination. To support this measurement, we sent text as a data input through our system. The output of receiver is equalized data and compare with data input sent. From this experiment, we get bit error percentage of $3 \%$ for $2 \mathrm{~m}$ distance, $7.1 \%$ for $5 \mathrm{~m}$ distance, and $12.8 \%$ for $7 \mathrm{~m}$ distance from scale of $100 \%$. 


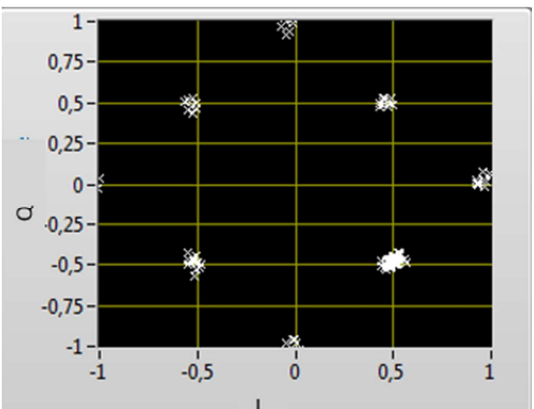

(a)

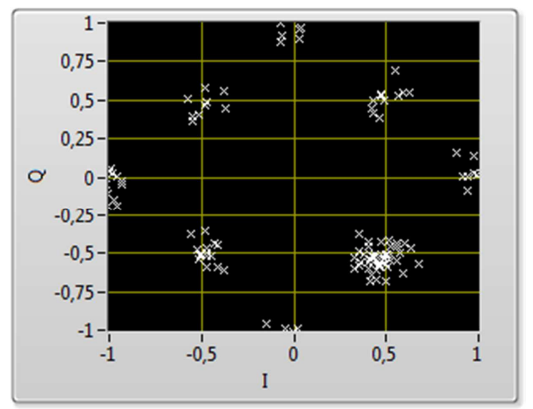

(b)

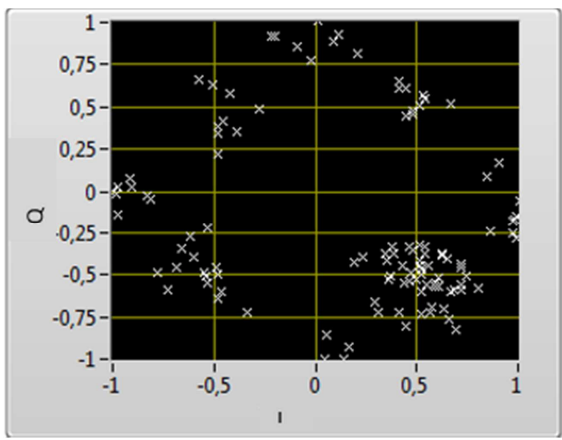

(c)

Figure 14. Star Constellation with MMSE Equalizer (a) 2m (b) 5m (c) 7m

\subsection{Measurement for Circular Constellation with ZF equalizer}

In this section, we implement and measure the combination of Circular constellation with ZF equalizer. The first measurement observes the diagram constellation of this combination with $2 \mathrm{~m}, 5 \mathrm{~m}$, and $7 \mathrm{~m}$ distance between transmitter and receiver. The figure 15 shows the constellation diagram of this combination with distance variation. When using $2 \mathrm{~m}$ distance is gotten the best result constellation, while the distance above $2 \mathrm{~m}$ is more scattered. The second measurement investigates the performance of this combination. Therefore, we sent text as a data input through our system. The output of receiver is equalized data and we compare it with data input. From this experiment, we get bit error percentage of $3 \%$ for $2 \mathrm{~m}$ distance, $6.8 \%$ for $5 \mathrm{~m}$ distance, and $11 \%$ for $7 \mathrm{~m}$ distance from scale of $100 \%$. 


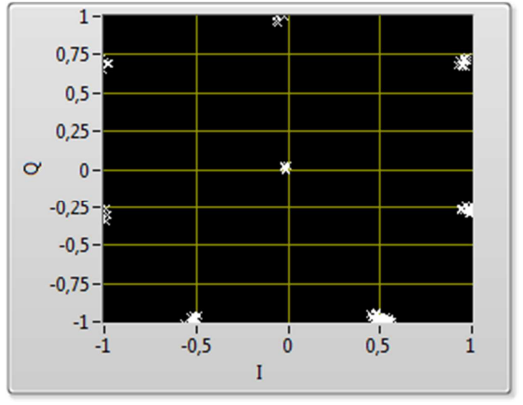

(a)

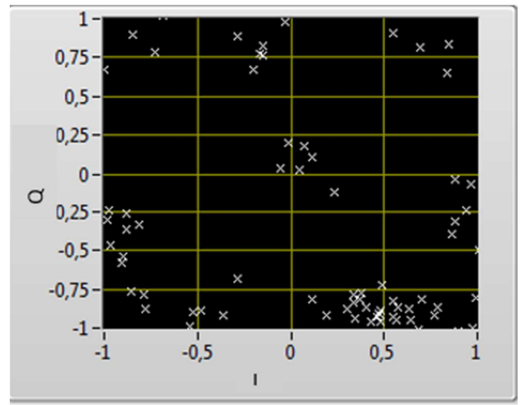

(b)

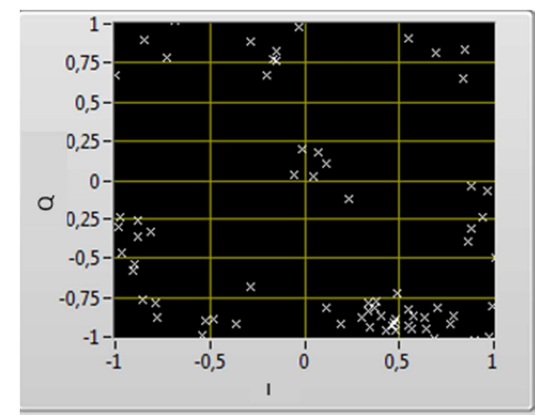

(c)

Figure 15. Circular Constellation with ZF Equalizer (a) 2m (b) 5m (c) 7m

\subsection{Measurement for Circular Constellation with MMSE equalizer}

We implement 8-QAM modulation and circular constellation both in transmitter and receiver, and also implement MMSE equalizer in receiver. The first measurement is to observe the constellation diagram with $2 \mathrm{~m}, 5 \mathrm{~m}$, and $7 \mathrm{~m}$ distance between transmitter and receiver. The figure 16 shows constellation diagram with distance variation. When using $2 \mathrm{~m}$ distance, the best performance is achieved, while the distance above $5 \mathrm{~m}$, the constellation is scattered. The second measurement is to investigate the performance of this combination. To support this measurement, we send text as a data input through our system. The output of receiver is equalized data and we compare it with data input. From this experiment, we get bit error percentage of $2 \%$ for $2 \mathrm{~m}$ distance $5.6 \%$ for $5 \mathrm{~m}$ distance, and $10 \%$ for $7 \mathrm{~m}$ distance from scale of $100 \%$. 


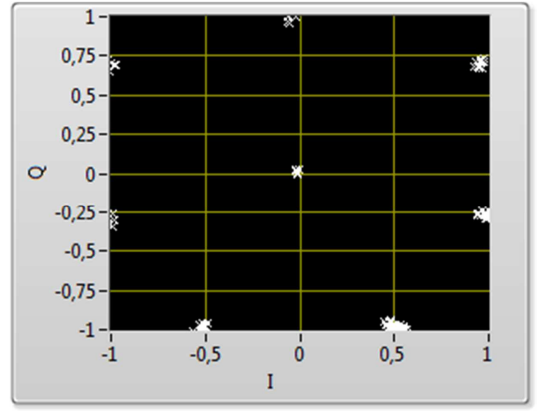

(a)

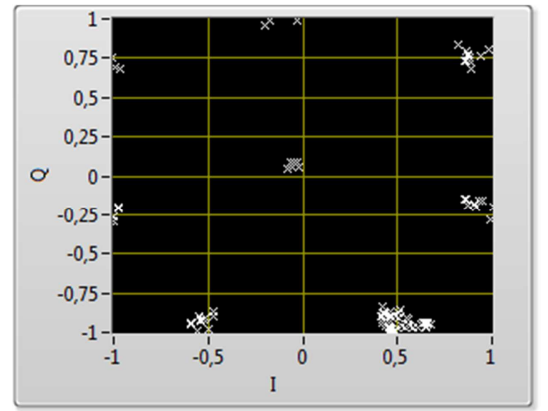

(b)

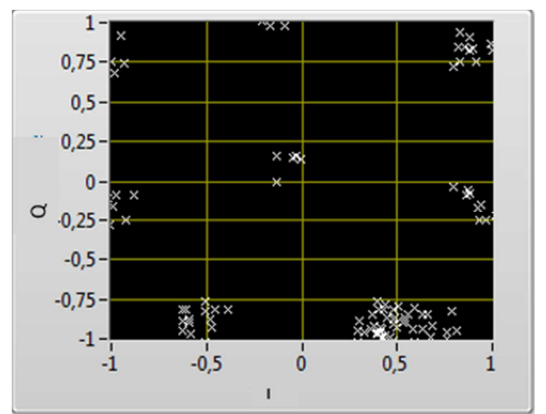

(c)

Figure 16. Circular Constellation with MMSE (a) 2m (b) $5 \mathrm{~m}$ (c) $7 \mathrm{~m}$

\subsection{Measurement Bit Error Performance}

In this section, we present a bit error comparison graph for overall measurement. In this measurement we use text file "Politeknik Elektronika Negeri Surabaya", in order to calculate the bit error of this text, we extract into binary code and compare the text sent by the transmitter and text received by the receiver. Figure 17 shows the bit error comparison between square-ZF, star-ZF, circular-ZF, square-MMSE, star-MMSE, and circularMMSE. From the higher of the scale number indicates the more error received.

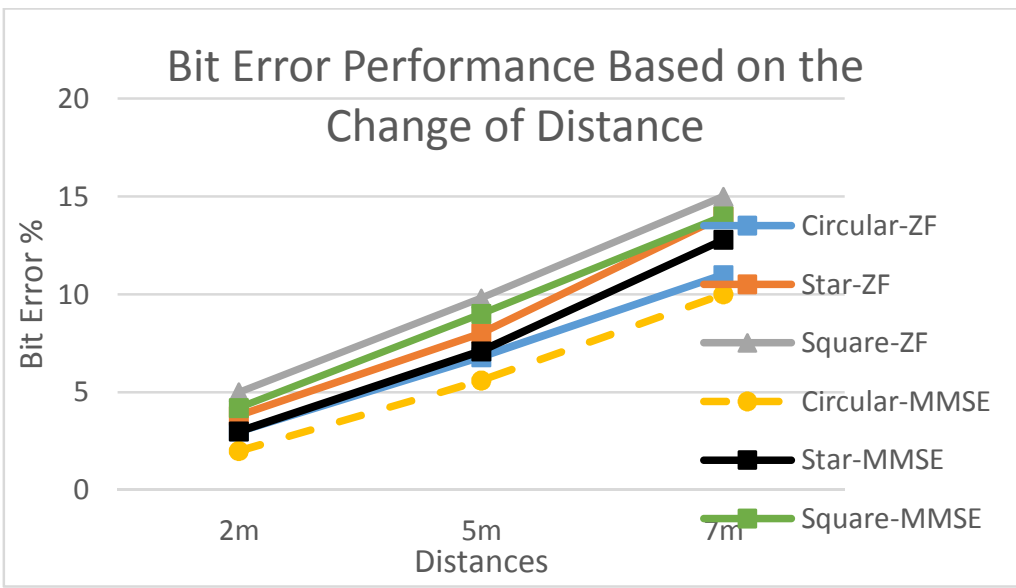

Figure 17.Bit Error Performance Based on the Change of Distance 
From the figure 17 we know that the combination of circular with ZF equalizer gets better performance than the other combination with ZF equalizer such as star and square with $3 \%, 6.8 \%$, and $11 \%$ error for $2 \mathrm{~m}, 5 \mathrm{~m}$, and $7 \mathrm{~m}$ distance. When we combine with MMSE equalizer technique, the system gets the best performance. The combination of circular with MMSE technique has better performance, which is about 1\%, than the combination of circular with ZF technique, because this system has signal to noise ratio estimation process. Therefore, the MMSE is not only mitigate the effect of ISI, but also it minimizes the noise power.

\section{CONCLUSION}

We have presented the performance of the circular 8-QAM and MMSE equalizer combination using USRP as hardware front end device. The experiments have shown that the combination of circular and MMSE equalizer get better performance than others, for $2 \mathrm{~m}$ distance circular-MMSE get $2 \%$ error and circular-ZF get $3 \%$ of error; however the performance decrease when the distance is increased. From the result of experiment above we know that MMSE equalizer has better performance, than $\mathrm{ZF}$ equalizer because the MMSE algorithm is more complex than $\mathrm{ZF}$ and also MMSE not only mitigate the effect of ISI, but also minimize the noise power. The distance between transmitter and receiver is greatly affecting the performance of our proposed system. The higher distance between transmitter and receiver make the performance of system decrease.

\section{Acknowledgements}

This research is granted by Politeknik Elektronika Negeri Surabaya (PENS) Fresh graduate Scholarship for Master's Degree Students.

\section{REFERENCES}

[1] Bernard Sklar. Digital Communications: Fundamentals and Applications (2 edition). New Jersey: Prentice Hall PTR, 2001.

[2] Laia Nadal, Josep Maria Fàbrega, Javier Vílchez, and Michela Svaluto Moreolo, Experimental Analysis of 8-QAM Constellations for Adaptive Optical OFDM Systems, IEEE PHOTONICS TECHNOLOGY LETTERS, VOL. 28, NO. 4, FEBRUARY 15, 2016

[3] Y. Li, S. Xu, and H. Yang, "Design of Circular Signal Constellations in the Presence of Phase Noise," in Proc. Int. Conf. on Wireless Communication.,Networking and Mobile Computing, Oct. 2008

[4] Pollet, T., Spruyt, P., Moeneclaey, M., "The BER performance of OFDM systems using non-synchronized sampling," Proc. IEEEGLOBECOM, vol.1, pp.253-257, 1994

[5] Nisha Achra, Garima Mathur, Prof. R.P. Yadav, Performance Analysis of MIMO OFDM System for Different Modulation Schemes under Various Fading Channels, International Journal of Advanced Research in Computer and Communication EngineeringVol. 2, Issue 5, May 2013 
[6] Abirami M, Gandhiraj R, Soman K. P, Performance Analysis of Real Time OFDM Based Communication System Using GNU Radio and USRP, International Journal of Advanced Research in Computer Scienceand Software Engineering, ISSN : 2277 128X, Volume 3, June 2013

[7] A. Y. F. Youssef, K. M. H. Hassan, M. G. Mostafa, M. T. Saad, M.Khairy,Implementation of a wireless OFDM system using USRP2 and USRP N210 Kits,Graduation Project Report,Faculty ofEngineering,CairoUniversity,Giza,Egypt,July 2012

[8] Duc Toan Nguyen, Impelementation of OFDM System using USRP and GNU Radio, University of Wollonggong, 2013.

[9] R. Rios-Müller, J. Renaudier, P. Tran, G. Charlet, Experimental Comparison of Two 8-QAM Constellations at 200 Gb/s over Ultra Long-Haul Transmission Link, ECOC 2014, Cannes - France

[10] A. Kanchan, S. Dwivedi, Comparison of BER Performance in OFDM Using Different Equalization Techniques, International Journal ofEngineering and Advanced Technology (IJEAT), ISSN:22498958,Volume-1, Issue-6, August 2012

[11] Beena R. Ballal, Ankit Chadha, Neha Satam, Orthogonal Frequency Division Multiplexing and its Applications, International Journal of Science and Research (IJSR), India Online ISSN: 2319-7064, Volume 2 Issue 1, January 2013

[12] Quadrature Amplitude Modulation, http:en.wikipedia.org, accessible April 2016.

[13] J. G. Proakis, "Digital Communications," Fourth edition, New York: McGraw-Hill, 2001

[14] Michael McLaughlin, Comparison of 8-star QAM and other 8-point Constellations,IEEE P802.15 Working Group for Wireless Personal Area Networks (WPANs), 2008

[15] Dsplog.com, "MIMO-OFDM with Different Equalization", access at 10 June 2016 